\title{
High-quality global hydrogen silsequioxane contact planarization for nanoimprint lithography
}

\author{
Serkan Büyükköse ${ }^{\text {a) }}$ \\ NanoElectronics Group, MESA + Institute for Nanotechnology, University of Twente, P.O. Box 217, 7500 \\ AE Enschede, The Netherlands \\ Boris Vratzov \\ NT\&D-Nanotechnology and Devices, Wirichsbongardstr. 24, 52062 Aachen, Germany \\ Wilfred G. van der Wiel \\ NanoElectronics Group, MESA + Institute for Nanotechnology, University of Twente, P.O. Box 217, 7500 \\ AE Enschede, The Netherlands
}

(Received 21 October 2010; accepted 13 February 2011; published 11 March 2011)

\begin{abstract}
The authors present a novel global contact planarization technique based on the spin-on-glass material hydrogen silsequioxane (HSQ) and demonstrate its excellent performance on patterns of 70 $\mathrm{nm}$ up to several microns generated by UV-based nanoimprint lithography. The HSQ layer $(\sim 165 \mathrm{~nm})$ is spin coated on the imprinted organic layer and planarized by pressing it with a flat wafer at room temperature. Before retracting the planarization wafer, the HSQ is hardened by baking at 120 or $70{ }^{\circ} \mathrm{C}$, depending on the underlying material. Fluorine-based reactive ion etching (RIE) is used to etch the HSQ (etch-back) down to the top of the features in the organic imprint layer. Subsequently, oxygen-based RIE is used to etch the organic imprint layer in the exposed regions, thereby transferring the imprinted pattern down to the substrate. The etch selectivity between the HSQ and the underlying layers is found to be more than 1:100, enabling very accurate pattern transfer with excellent critical dimension control and well-defined undercut profile suitable for further metal liftoff processes. The dependence of the contact planarization quality on the HSQ spinning speed and pressure is investigated, achieving a global planarization degree as good as 93\%, an improvement of 45\% compared to standard spin-coating planarization. (C) 2011 American Vacuum Society. [DOI: 10.1116/1.3562939]
\end{abstract}

\section{INTRODUCTION}

In microfabrication and nanofabrication, sometimes global (wafer-scale) planarization is required, while in other cases only partial or local planarization is sufficient. Spin-onglass (SOG) is a widely used planarization material in semiconductors. SOG materials are siloxanes or silicates mixed in alcohol-based solvents and exhibit a good planarization capability. ${ }^{1}$ However, the degree of planarization (DOP) decreases with increasing spacing between features, ${ }^{2}$ and subsequent pattern transfer by reactive ion etching (RIE) is also limited by the design and spacing between features. In some cases, such as in multistep fabrication processes, high-quality planarization is also crucial and simple application of a SOG gives insufficient global planarization. Although there exist some improved SOG-based planarization techniques, they generally require more than one SOG layer or additional layers of different materials ${ }^{3}$ or additional etch processes. ${ }^{4}$

In this work, high-quality global contact planarization with only one layer of SOG was realized for parallel reproduction of patterns with dimensions in the micrometer and nanometer ranges. Contact planarization ${ }^{5}$ was obtained by applying force at room temperature onto the liquid planarization material via an optically flat template in a manner similar to hot embossing lithography. The planarization material

${ }^{a)}$ Electronic mail: s.buyukkose@utwente.nl is hardened via a thermal curing process and leaves a flat surface on the wafer after retraction of the planarization wafer. Importantly, this method is capable of achieving both excellent local and global planarization. Hydrogen silsequioxane (HSQ) was chosen as a SOG material because of its room-temperature deformation capability. Although HSQ is ordinarily used as a negative-tone electron-beam lithography (EBL) resist, experimental investigations showed that it can also be applied as a nanoimprint resist at room temperature. $^{6,7}$ Excellent etch resistance of HSQ under oxygen-based reactive ion etching is another advantage for pattern transfer. Critical dimension loss during etching of the organic residual layer that remains after UV-based nanoimprint lithography (NIL) can be prevented by using HSQ. As an etch mask, the thickness of the HSQ layer all over the substrate is critical. The specific aim of the planarization in this work was to reduce thickness variations in the HSQ layer on patterned and nonpatterned regions over the entire substrate.

In the literature there are some examples of contact planarization of patterned surfaces. ${ }^{8,9}$ Sun et al. used polymethylmethacrylate (PMMA) and novolak resin materials to planarize a surface, in contrast to our HSQ planarization method; they used relatively high temperature $\left(175^{\circ} \mathrm{C}\right)$ and pressure (up to 50 bar) to force the planarization materials to flow over the substrate. ${ }^{8}$ A different method offered planarization at room temperature of prepatterned surfaces us- 
(a)

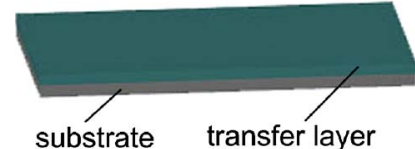

(c)

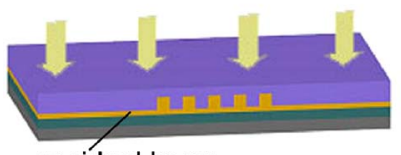

residual layer (b)

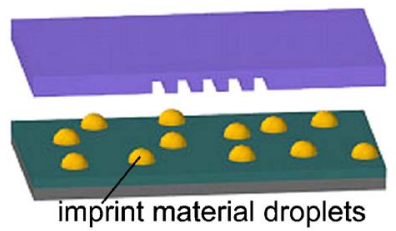

(d)

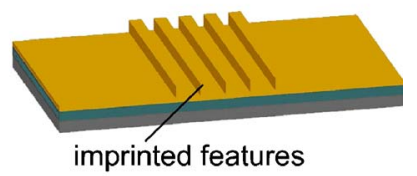

FIG. 1. (Color online) UV-based nanoimprint lithography: (a) First wafers were coated with an organic transfer layer (e.g., PMMA); (b) a UV-curable organic imprint material was dispensed on the surface locally adjusting the volume; (c) force was applied with a transparent template, and the imprint material was exposed to UV light; and (d) after removing the template, imprinted features were obtained on the surface.

ing a UV-based imprint process [step-and-flash imprint lithography (S-FIL)] and flat templates. ${ }^{9}$ In the latter study, the planarization on wafer scale is achieved in a step and repeat mode, where in every step a single imprint field is planarized. This die-by-die planarization method offers good results; however on a wafer scale it is very slow and critical for contaminations. In our case, HSQ enables a fast roomtemperature planarization process, and its high etch selectivity with respect to the underlying organic resists makes it a favorable material for planarization and dimension control. Even though in this work features are defined by UV-based nanoimprint lithography, it should be noted that this planarization method is very generic and can be also applied to any other structures defined by other lithography techniques or etching processes.

\section{EXPERIMENT}

Pattern fabrication on $\mathrm{Si}$ wafers was carried out using S-FIL ${ }^{\mathrm{TM}}$ on an Imprio $^{\mathrm{TM}} 55$ tool from Molecular Imprints, Inc. To obtain high-aspect-ratio features, $100 \mathrm{~mm}$ doublesided polished (DSP) Si wafers were first spin coated with a transfer layer and hot baked [see Fig. 1(a)]. DUV-30J (from Brewer Science, Inc.) which is normally applied as organic bottom antireflective coating, or PMMA was used as a transfer layer because of its low etching resistance compared to HSQ under oxygen plasma, and solubility of the PMMA in standard solvents (e.g., acetone) for potential liftoff applications. A 2 wt \% PMMA (Mw $350 \mathrm{kD})$ in toluene was prepared for our experiments. The PMMA itself was purchased from Sigma Aldrich, Inc. DUV-30J and PMMA were spin coated at $3000 \mathrm{rpm}$ and baked on a hot template at 150 and $120{ }^{\circ} \mathrm{C}$, respectively. The features were imprinted on top of the transfer layer into a low-viscosity, organic, and UVcurable material at room temperature and under low force $(2-3 \mathrm{~N})$. As an imprint material Monomat ${ }^{\mathrm{TM}}$ (from Moleculer Imprints, Inc.) was used. This imprint material was deposited by field-to-field dispensing and cured through the

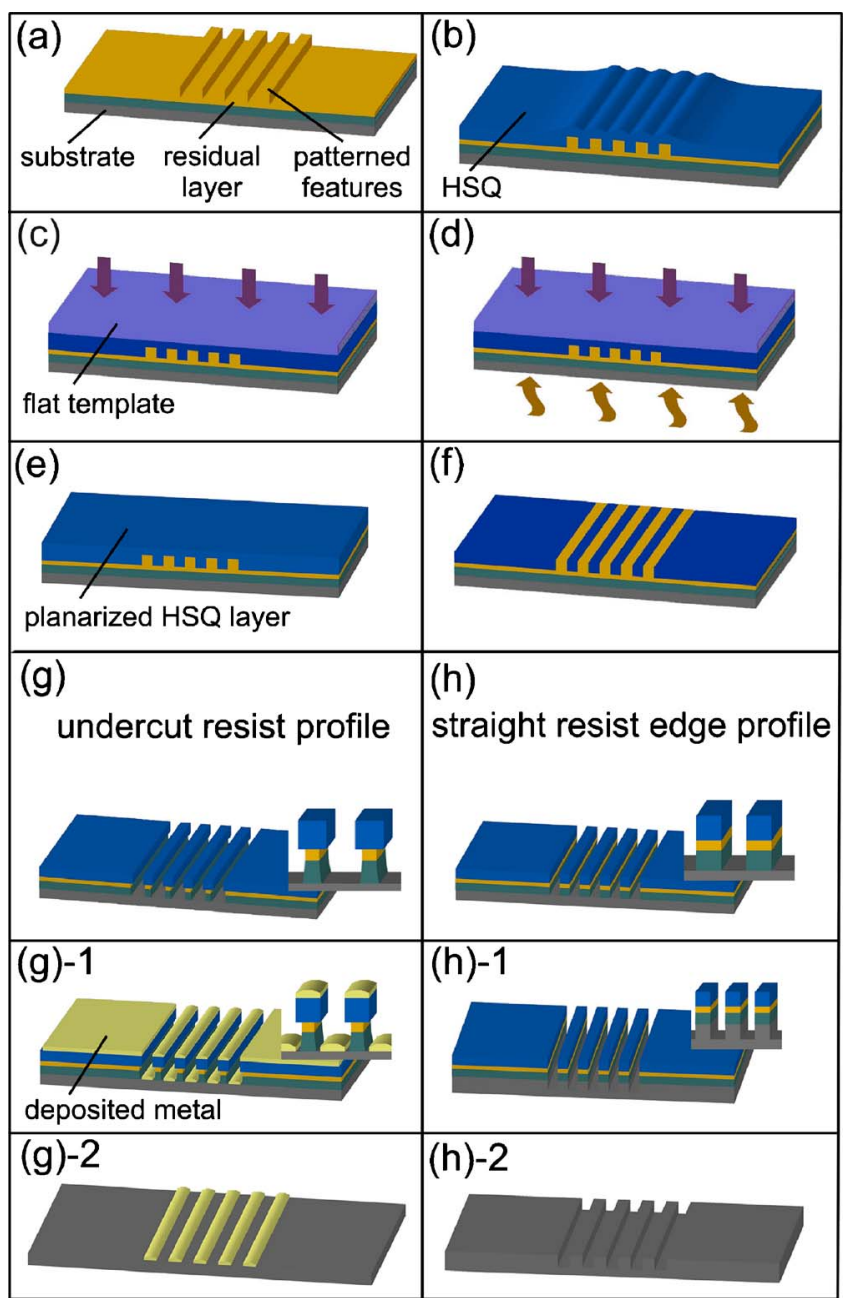

FIG. 2. (Color online) Planarization process steps for imprinted features as explained in the text: (a) features defined by UV-assisted nanoimprint lithography, (b) HSQ spin coating, (c) room-temperature contact planarization by a flat stamp, (d) HSQ heating at constant pressure, (e) contact-planarized surface, (f) etching-back HSQ overlayer. After step (f) two different process routes can be followed, depending on the fabrication requirements: $(\mathrm{g})$ pattern transfer with an undercut resist profile via oxygen-based RIE, (g)-1 metallization, (g)-2 transfer layer removal (e.g., by acetone in case of PMMA), and (h) straight resist edge profile, (h)-1 further etching into substrate, (h)-2 transfer layer removal.

transparent imprint template using broadband UV light (exposure dose of $80 \mathrm{~mJ} / \mathrm{cm}^{2}$ and $230-360 \mathrm{~nm}$ wavelength) [see Figs. 1(b) and 1(c)]. After S-FIL, the residual imprint material and transfer layer remained on the surface, and for further pattern transfer they need to be etched. A schematic overview of the process is given in Fig. 2.

The imprint templates (molds) used for the experiments were fabricated by standard EBL and RIE techniques with a $25 \times 25 \mathrm{~mm}^{2}$ active area (imprint area), consisting of different test features such as line and spaces, dots, or holes with dimensions from $60 \mathrm{~nm}$ up to $500 \mathrm{~nm}$ and pitch sizes varying from 1:1 up to 1:5. HSQ, XR-1541 6\% with carrier solvent methylisobutylketone, from Dow Corning, Inc., was deposited on top of the imprinted layer by spin coating [see Fig. 2(b) ]. In contrast to our work, if a thermoplastic planarization material is used, the planarization process has to be per- 
formed at higher temperature than the glass transition temperature $\left(T_{g}\right)$ to make the material less viscous. However, in our case, heating does not make HSQ softer, but instead makes it harder to be deformed under pressure. Therefore, planarization was performed at room temperature and HSQ was not thermally treated after spin coating. For the contact planarization, a blank $100 \mathrm{~mm}$ DSP Si wafer was used as the flat template [see Figs. 2(c) and 2(d)]. Prior to planarization, the flat $\mathrm{Si}$ template was treated with an antisticking release layer to facilitate an easy separation of the template from the HSQ layer. $1 \mathrm{H}, 1 \mathrm{H}, 2 \mathrm{H}, 2 \mathrm{H}$-perfluorodecyltrichlosilane was deposited on the flat $\mathrm{Si}$ template via gas-phase evaporation method under vacuum. ${ }^{10} \mathrm{~A}$ hot embossing NIL tool (from Obducat) was used to press the flat Si template onto the HSQ coated wafers at room temperature; in this tool pressure is applied by means of compressed air, ensuring pressure uniformity over the entire imprint area (Soft Press ${ }^{\circledR}$ Technology).

After getting a planarized surface [see Fig. 2(e)], the etchback of the overlayer of HSQ [see Fig. 2(f)] and pattern transferring through the residual imprint material and transfer layer that remained after S-FIL [see Fig. 2(g)] were performed under $\mathrm{CHF}_{3}$ plasma and $\mathrm{O}_{2}$ plasma, respectively, in an Electrotech PF 340 apparatus. Cross-sections of samples were examined by a LEO 1550 high-resolution scanning electron microscope (HR-SEM), and topographical data were obtained using a DI 3100a Veeco atomic force microscope (AFM).

\section{RESULTS AND DISCUSSION}

Several imprinted line-shaped features with different widths from sub-100 nm to $500 \mathrm{~nm}$ and separation from 100 $\mathrm{nm}$ to $2.5 \mu \mathrm{m}$ were prepared for planarization experiments. The height of the features was around $100-150 \mathrm{~nm}$. The transfer layer thickness was $\sim 60 \mathrm{~nm}$ for DUV-30J and $\sim 120 \mathrm{~nm}$ for PMMA. A homogenous residual layer of organic imprint material was also found around $60 \mathrm{~nm}$ after imprinting. In order to perform good planarization, the planarization layer needs to be as thick as possible. On the other hand, in order to have a well-controlled back-etch-step with an appropriate stop at the top of the imprinted features, we aimed at an as thin as possible overlayer of HSQ. Therefore, as a first step we have investigated the spin-coating dynamics of HSQ on blank Si wafers for different spin rotation speeds. Figure 3 shows the HSQ thickness versus rotational speed during spin coating, varying from $500 \mathrm{rpm}$ up to $3500 \mathrm{rpm}$ in $500 \mathrm{rpm}$ steps. For 1500 and $2000 \mathrm{rpm}$, the thicknesses are around $135 \pm 3$ and $115 \pm 2 \mathrm{~nm}$, respectively, and show good uniformity over the substrate. For $500 \mathrm{rpm}$, the HSQ film lost its thickness uniformity on the wafer. It seems that these thicknesses are sufficient to fill the trenches. However, the overlayer thickness (the thickness of HSQ on top of the features) is also critical for contact planarization. Especially, in the case of largely separated features, there has to be sufficient material accumulated on the elevated features to be transferred toward the gaps between the features during the application of pressure. For $1000 \mathrm{rpm}$, the coating thickness

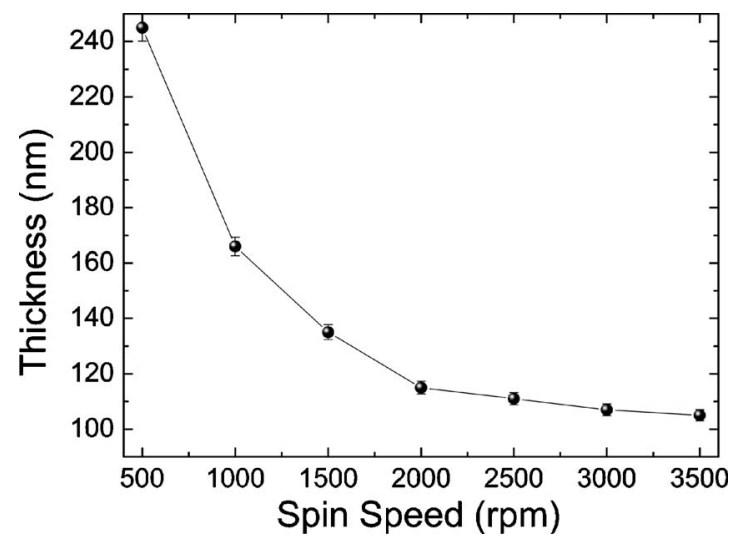

FIG. 3. HSQ layer thickness vs spin rotation speed.

was found to be $166 \pm 3 \mathrm{~nm}$ while keeping the coating uniformity. We therefore used $1000 \mathrm{rpm}$ as the rotational speed during spin coating.

As mentioned above, with only spin coating, HSQ is able to give suitable results for features with low height $(\sim 40 \mathrm{~nm})$ and high feature density $(\sim 65 \%),{ }^{11}$ where the feature density is defined as [linewidth/(linewidth + spacing)]. Nevertheless, when the feature height increases or the feature density decreases, additional steps are required to obtain adequate planarization. In our case, the feature height is around $100-150 \mathrm{~nm}$ and feature densities vary from $50 \%$ to $17 \%$.

Figure 4 shows a sample, only spin coated with HSQ and baked at $120^{\circ} \mathrm{C}$, without any additional steps. It can be seen that planarization degrades when the feature size and separation increase, giving a maximum of $\sim 50 \mathrm{~nm}$ height difference. Even for high-density features there is still no complete local planarization.

To study the pressure dependence, a number of contact planarization experiments were performed with HSQ spincoated samples at room temperature under pressures of 10 , 20 , and 40 bar. Afterward, the temperature was increased to $120{ }^{\circ} \mathrm{C}$ for $2 \mathrm{~min}$ in order to increase the HSQ hardness without removing the template. Figure 5 shows the depen-

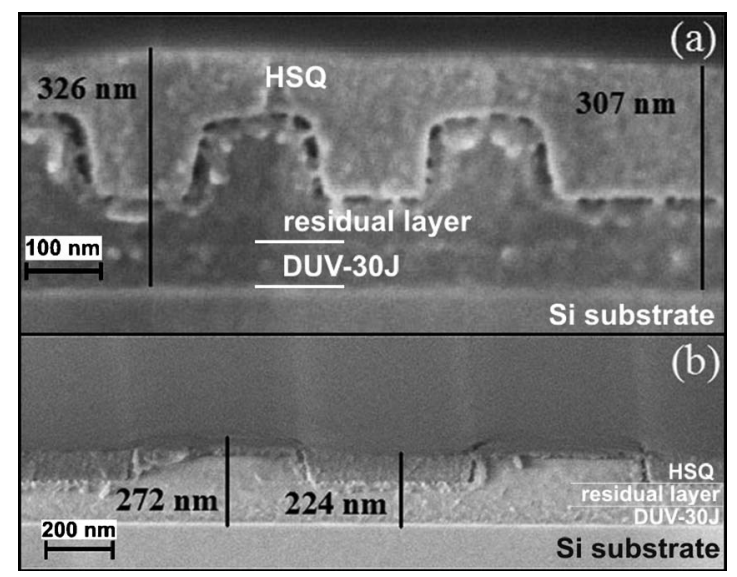

FIG. 4. HR-SEM pictures of HSQ spin-coated samples with (a) $100 \mathrm{~nm}$ linewidth and $100 \mathrm{~nm}$ period, and (b) $500 \mathrm{~nm}$ linewidth and $500 \mathrm{~nm}$ period. 


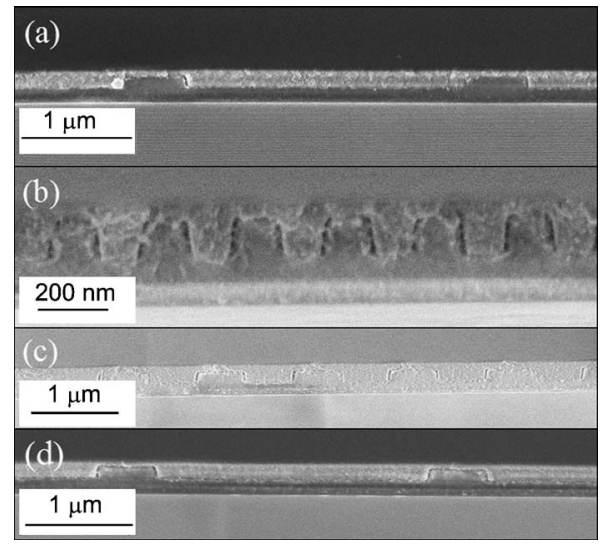

FIG. 5. HR-SEM pictures of contact-planarized samples (HSQ imprint material and DUV-30J transfer layer) with [(a) and (b)] 40, (c) 20, and (d) 10 bar pressures applied to the flat stamp.

dence of the HSQ planarization on the applied pressure. There is no significant difference between the results for different pressures, and HSQ redistribution from overlayer to cavity between features is still possible even at 10 bar.

A quantitative measurement of the step height reduction was performed using AFM. Planarization was performed under 40 bar on spin-coated samples without prebaking. To demonstrate the quality of planarization, a parameter known as the planarization factor can be defined as $\beta=1-t_{f} / t_{i}$, where $t_{i}$ and $t_{f}$ are the initial and final step heights, respectively [see Fig. 6(a) inset]. The "degree of planarization" is given by $100 \beta$. Figure 6(a) shows the surface profiles of 100 -nm-wide lines and $200 \mathrm{~nm}$ spacing at different stages, after imprinting, spin coating, and planarization, where $\beta_{p}$ is the planarization factor after contact planarization and $\beta_{s}$ is the planarization factor after spin coating, but before contact planarization. The initial HSQ layer thickness was around $165 \mathrm{~nm}$. The DOP increased from $88 \%$ to $95 \%$ by contact planarizing the sample with the flat template. Figure 6(b) also shows the planarization degree for features with 100 and $500 \mathrm{~nm}$ linewidths for different spacing values (varying from $100 \mathrm{~nm}$ to $2.5 \mu \mathrm{m}$ ). A DOP of around $95 \%$ can be accomplished for all feature sizes and spacing. It should be noted that the surface profiles were taken in the close vicinity of the featured area; in other words, Figs. 6(a) and 6(b) give information about local planarization on the surface.

To demonstrate global planarization, high-resolution SEM pictures from patterned and nonpatterned regions, $2-3 \mathrm{~mm}$ away from each other, were taken for both contact-planarized and nonplanarized samples. Further distances, more than 2-3 $\mathrm{mm}$, have already the same value because of the spin-coating uniformity. Figures 7(a) and 7(b) show nearly 64\% DOP after HSQ spin coating. However, after contact planarization, the global DOP increased to 93\% [see Figs. 7(c) and 7(d)]. This DOP value means uniform HSQ thickness over the sample after the etch-back process, and that certain regions on the wafer can be prevented from being exposed by ionic plasma. In other words, HSQ is not only an etch mask for featured areas, but also an etch mask for nonfeatured areas.
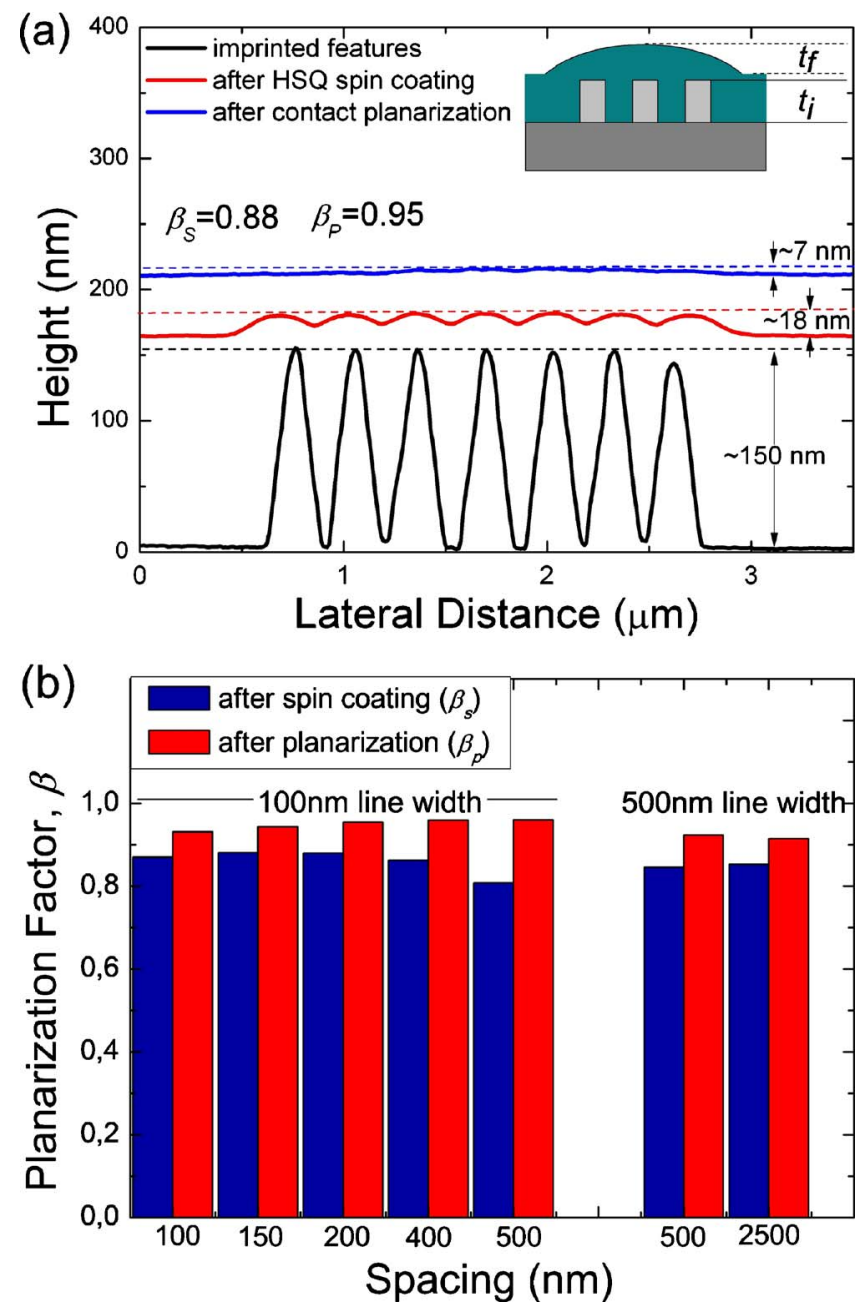

FIG. 6. (Color online) (a) Surface profiles of imprinted, HSQ spin coated, and contact-planarized samples and definition of $t_{i}$ and $t_{f}$ (inset) and (b) planarization factors for different pattern sizes before $\left(\beta_{s}\right)$ and after $\left(\beta_{p}\right)$ contact planarization.

As indicated in Fig. 2, for pattern transferring it is also possible to get perpendicularly etched sidewalls by optimizing etching parameters in addition to undercut sidewalls. Although in our work it is specifically aimed to get undercut profiles after pattern transferring for metallization and liftoff purposes, an example of perpendicular pattern transfer is also given to demonstrate the capability of this general-purpose method (see Fig. 8).

For further pattern transferring for liftoff, the contactplanarized HSQ overlayer was etched back for 3 min under $\mathrm{CHF}_{3}$ plasma with $40 \mathrm{~W}$ rf power, $25 \mathrm{sccm}$ gas flow, and 10 mTorr chamber pressure. The organic imprint layer etching and transfer layer etching were performed under $\mathrm{O}_{2}$ plasma with $20 \mathrm{~W}$ rf power, $20 \mathrm{sccm}$ gas flow, and $10 \mathrm{mTorr}$ chamber pressure. Figures 9 and 10 show HR-SEM pictures of etched samples with different feature sizes. While the DUV30J layer coated samples were cured at $120^{\circ} \mathrm{C}$ under pressure after the planarization, the PMMA coated samples were cured at $70{ }^{\circ} \mathrm{C}$ to prevent possible damage to the PMMA layer because of its $T_{g}$ between 85 and $165^{\circ} \mathrm{C}$. The suitabil- 


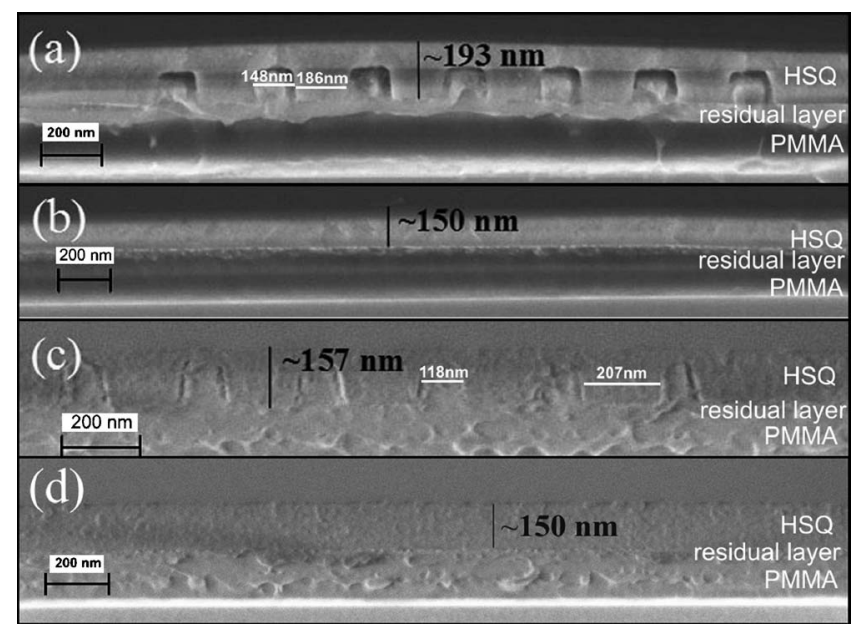

FIG. 7. HR-SEM pictures of patterned and unpatterned regions on the wafer. (a) and (b) shows patterned and unpatterned regions after spin coating, and (c) and (d) show patterned and unpatterned regions after contact planarization. Mold feature period is $337.5 \mathrm{~nm}$ (linewidth:spacing=1:1.25) for (a) and $320 \mathrm{~nm}$ (linewidth: spacing $=1: 1.8)$ for $(\mathrm{c})$

ity of HSQ as a hard etching mask, due to the etch selectivity higher than 1:100 between HSQ and PMMA under $\mathrm{O}_{2}$ plasma, is clearly observed in both Figs. 9 and 10. Very accurate transfer of $100 \mathrm{~nm}$ lines into the PMMA with $30 \mathrm{~nm}$ underetched profile on each side clearly indicates critical dimension control capability of our method. The final pattern transfer was performed by metal deposition and liftoff process. Therefore, an adhesion layer of $5 \mathrm{~nm} \mathrm{Cr}$ followed by a $30 \mathrm{~nm} \mathrm{Au}$ layer on top of it was deposited using electronbeam evaporation and was lifted off in acetone. Figure 11 shows $35-\mathrm{nm}$-thick $\mathrm{Au} / \mathrm{Cr}$ metal grating with $123 \mathrm{~nm}$ linewidth and $120 \mathrm{~nm}$ spacing after bilayer metal deposition and liftoff process. For the initial definition of the features shown in Fig. 11, an imprint template consisting of a grating with $120 \mathrm{~nm}$ linewidth and $120 \mathrm{~nm}$ spacing was used that demonstrates the high critical dimension control of our whole process cycle.

\section{SUMMARY AND CONCLUSIONS}

It has been shown that global contact planarization with HSQ can be achieved at room temperature with a flat and blank template. This technique has shown an improvement of $\sim 45 \%$ compared to standard spin-coating planarization.

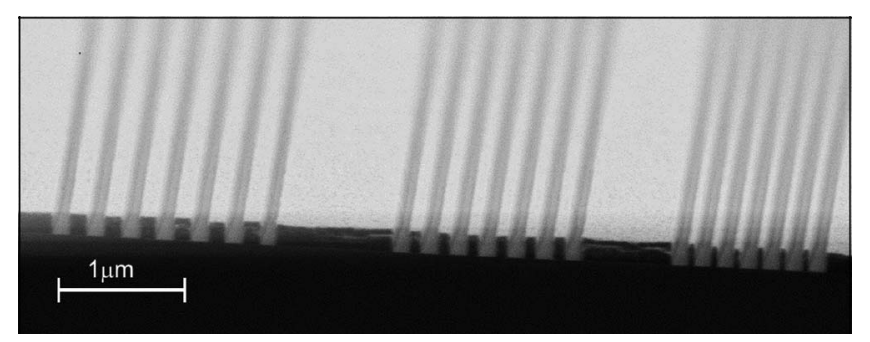

FIG. 8. Perpendicular pattern transfer into the residual and DUV-30J transfer layer according to Fig. 2(h). Imprinted features have 150, 100, and $50 \mathrm{~nm}$ linewidths from left to right (all $125 \mathrm{~nm}$ spacing).

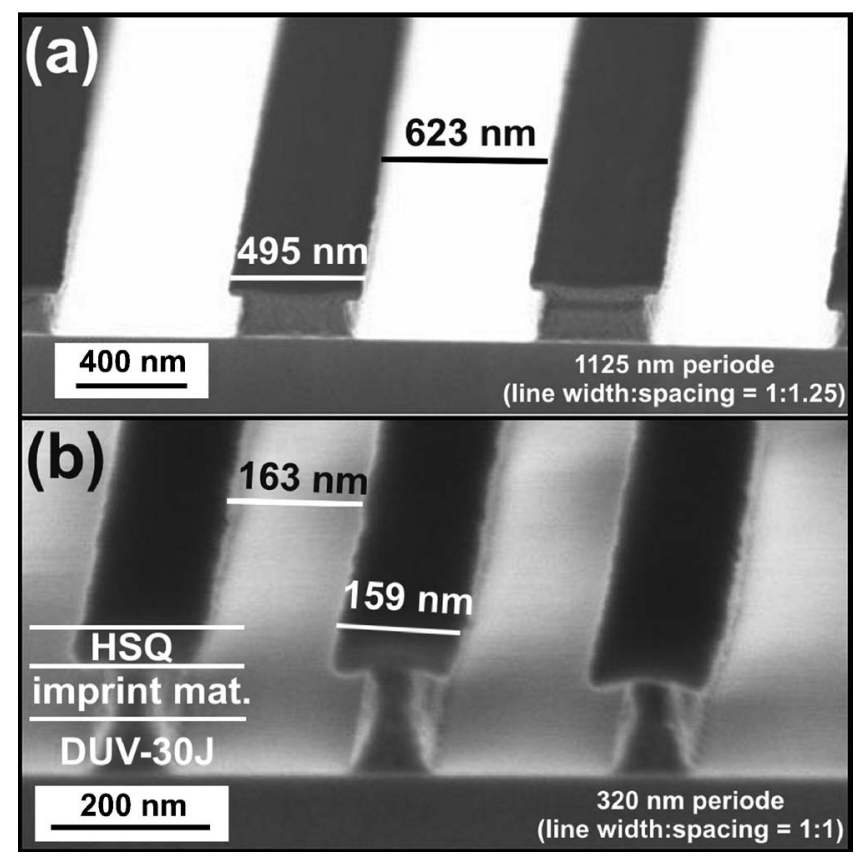

FIG. 9. HSQ/imprint material $(\sim 60 \mathrm{~nm}) / \mathrm{DUV}-30 \mathrm{~J}(\sim 60 \mathrm{~nm})$ layer coated samples etched under $\mathrm{CHF}_{3}$ plasma for $4.2 \mathrm{~min}$, followed by $\mathrm{O}_{2}$ plasma for $2 \mathrm{~min}$. After $\mathrm{CHF}_{3}$ plasma etching, the HSQ thickness is $\sim 40 \mathrm{~nm}$; (a) mold feature period is $1125 \mathrm{~nm}$ (linewidth: spacing $=1: 1.25)$ and (b) mold feature period is $320 \mathrm{~nm}$ (linewidth: spacing $=1: 1$ ).

Because of the inorganic nature of HSQ, HSQ shows excellent etch selectivity with respect to organic underlying layers. This was demonstrated by pattern generation with UVbased NIL and RIE processes. Very precise control of the critical dimensions and well-defined undercut resist profiles were obtained, suitable for metal deposition followed by liftoff. HSQ contact planarization is a generic method and can

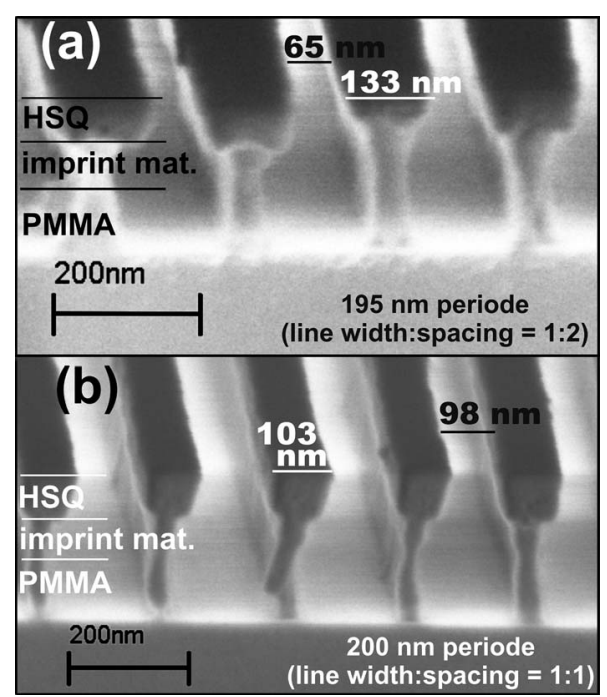

FIG. 10. HSQ/ImprintMat $(\sim 60 \mathrm{~nm}) / \mathrm{PMMA}(\sim 100 \mathrm{~nm})$ layer coated samples etched under $\mathrm{CHF}_{3}$ plasma for 3 min and following $\mathrm{O}_{2}$ plasma for $2 \mathrm{~min}$. After $\mathrm{CHF}_{3}$ plasma etching, the HSQ thickness is $\sim 75 \mathrm{~nm}$; (a) mold feature size is $195 \mathrm{~nm}$ period grating (65 nm 1:2 lines and spacing) and (b) mold feature size is $200 \mathrm{~nm}$ period grating (100 nm 1:1 lines and spacing). 


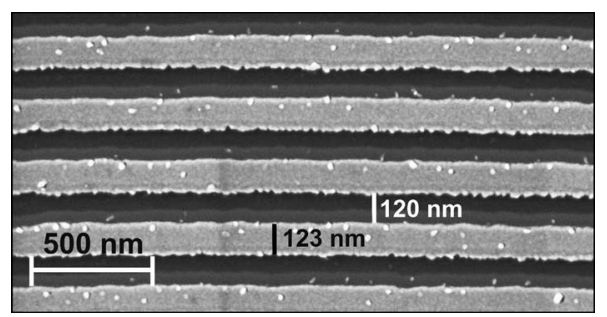

FIG. 11. SEM micrograph of metal grating with $123 \mathrm{~nm}$ linewidth and 120 $\mathrm{nm}$ spacing made by evaporation of 30-nm-thick gold layer on top of $5 \mathrm{~nm}$ $\mathrm{Cr}$ as adhesion layer and followed by liftoff process. The features on the initial imprint mold used for the pattern definition were $120 \mathrm{~nm}$ lines with $120 \mathrm{~nm}$ spacing.

therefore also be applied for other lithography techniques and multilayer applications because of its simplicity, fastness, low cost, and efficiency.

\section{ACKNOWLEDGMENTS}

The authors would like to thank Pieter F. Moonen for experimental help and PMMA preparation, Regina Luttge and Jurriaan Huskens for useful discussions, and Mark
Smithers for the HR-SEM work. This research was performed within the NanoArrays project financed by The Netherlands Technology Foundation STW.

${ }^{1} \mathrm{~S}$. Wolf, Silicon Processing for the VLSI Era-Process Integration (Lattice, California, 1990), Vol. 2, p. 229.

${ }^{2}$ D. L. W. Yen and G. K. Gopal, Proceedings of the 5th International IEEE VMIC Conference (IEEE, New York, 1998).

${ }^{3}$ G. Subramania, Nanotechnology 18, 035303 (2007).

${ }^{4}$ S. Ramaswami and A. Nagy, J. Electrochem. Soc. 139, 591 (1992).

${ }^{5}$ W.-S. Shih, J. Yota, H. Ly, K. Itchhaporia, and A. Smith, Technical Digest of the CS MANTECH Conference (CS Mantech, Beaverton, OR, 2007). ${ }^{6}$ Y. Igaku, S. Matsui, H. Ishigaki, J. Fujita, M. Ishida, Y. Ochiai, H. Namatsu, M. Komuro, and H. Hiroshima, Jpn. J. Appl. Phys., Part 1 41, 4198 (2002).

${ }^{7}$ K. Nakamatsu and S. Matsui, in Nanomanufacturing Handbook, edited by A. Busnaina (CRC, Boca Raton, 2007), Vol. 2, pp. 162-180.

${ }^{8}$ X. Sun, L. Zhuang, W. Zhang, and S. Y. Chou, J. Vac. Sci. Technol. B 16, 3922 (1998).

${ }^{9}$ S. V. Sreenivasan, A. McMackin, F. Xu, D. Wang, and N. Stacey, Micro Magazine 23, 37 (2005).

${ }^{10}$ P. Maury, M. Peter, V. Mahalingam, D. N. Reinhoudt, and J. Huskens, Adv. Funct. Mater. 15, 451 (2005).

${ }^{11}$ Y. Yoon, C. Choi, Y. Oh, D. Hong, S. Jin, and F. E. Talke, IEEE Trans. Magn. 45, 3527 (2009). 